\title{
Blood Flow through a Stenosed Artery with the Effect of Transverse Magnetic Field using a Non-Newtonian Model
}

\author{
G. C. Hazarika \\ Department of Mathematics \\ Dibrugarh University, Assam, India
}

\author{
Aparajita Sarmah \\ Department of Mathematics \\ Dibrugarh University, Assam, India
}

\begin{abstract}
One-dimensional, steady, Herschel-Bulkley fluid flow of blood through a stenosed artery under the effect of external magnetic field is studied. The blood is assumed as incompressible. The governing equations are solved analytically. This model has been used to study the influence of yield stress on blood flow through the stenosed artery. The effects of magnetic field on axial velocity, flow rate and wall shear rate has been shown graphically. The effects of all the parameters are quite significant on axial velocity, flow rate and wall shear rate as evidence from the results.
\end{abstract}

\section{Keywords}

Stenosis, yield stress, Herschel-Bulkley fluid flow.

\section{INTRODUCTION}

Blood is a suspension of formed cellular elements that includes red blood cells, white blood cells and platelets. Viscous liquids including whole blood can be divided in terms of rheological properties into Newtonian and general nonNewtonian and viscoelastic fluids. At low shear rate blood exibit yield stress and shows Non-Newtonian characteristics [1], [2], [3]. Also at low shear rate $(0.1$ per sec) the blood exibit yield stress and behaves like a Herschel Bulkley fluid[4], [5], [6]. Herschel Bulkley fluid is of general type and the results obtained by this model enables one, in deriving the corresponding results in cases of Newtonian fluid, Bingham plastic and power law fluid model by inserting appropriate measures of flow parameters and flow characteristics. Abnormal constriction of an artery is termed as stenosed artery. Existing literature in blood flow reveals that the shear rate of blood is low in stenosed artery. With the help of Magnetohydrodynamics the flow of blood through such type of arteries can be controlled. The applied magnetic field reduces the shear stress parameter and changes the speed of blood [7], [8], [9]. Some researchers have studied the flow of blood through a stenosed artery with slip at the wall wherein the rheology of blood is described by Herschel-Bulkley constitutive equation [10].

Here an attempt has been made to study the effects of magnetic fields in transverse direction. In doing so we shall stress upon the variation of axial velocity, wall shear stress, volumetric flow rate for different flow parameters.

\section{MATHEMATICAL FORMULATION}

\subsection{Assumptions}

Consider steady, laminar flow of blood through a straight, rigid tube of circular cross-section with the formation of a stenosis at the inner most vessel wall. The geometry of the stenosis is as shown is as shown in the Fig. a. The blood has been assumed to behave as Herschel -Bulkley fluid. Let us take the flow of blood as axially symmetric and fully developed (i.e. the flow in z-direction only). An external magnetic field of strength $B_{0}$ is applied in transverse direction.

\subsection{Flow Geometry}

It is assumed that the stenosis is developed in an axially symmetric manner. The radius of the artery $R(z)$ in stenosed region can be taken as [10]

$$
\begin{aligned}
R(z)= & R_{0}-\frac{\delta}{2}\left\{1+\operatorname{Cos} \frac{2 \pi}{L_{0}}\left(z-d-L_{0}\right)\right\} \\
& \left(d \leq z \leq d+L_{0}\right) \\
= & R_{0} \quad \text { (otherwise) }
\end{aligned}
$$

where $R_{0}$ and $R(z)$ are the radius of the uniform and constricted region. $L$ is the length of the artery, $L_{0}, d, \delta$ are the length, location and maximum height of the stenosis. And $r$ and $z$ are the radial and axial co-ordinates.

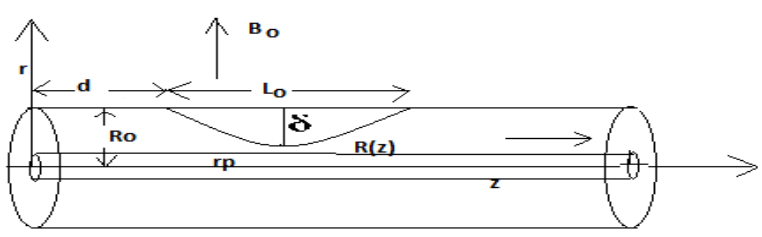

Fig a: flow geometry and co-ordinate system

\subsection{Governing Equations}

Under the applied magnetic field the governing equations of motion for laminar, incompressible, fully developed, one dimentional flow of blood is given by

$$
\left.\begin{array}{l}
0=-\frac{\partial p^{*}}{\partial z^{*}}+\frac{1}{r^{*}} \frac{\partial}{\partial r^{*}}\left(r^{*} \tau^{*}\right)-\sigma B_{0}{ }^{2} w^{*} \\
0=-\frac{\partial p^{*}}{\partial r^{*}}
\end{array}\right\}
$$

where $\tau$ represents the shear stress of blood behaving as a Herschel-Bulkley fluid and $p$ is pressure at any point.

The constitutive equation for Herschel -Bulkley fluid may be written as 
$-\frac{\partial w}{\partial r}=f(\tau)=\left\{\begin{array}{l}\frac{1}{k}\left(\tau-\tau_{H}\right)^{n}, \tau \geq \tau_{H} \\ 0, \tau \leq \tau_{H}\end{array}\right.$

where $w$ stands for the axial velocity and $\tau_{H}(\geq 0)$ is the yield stress and $k$, the fluid viscosity and $n(<=>1)$ is the fluid behavior index.

The boundary condition to the problem under study

$w^{*}=w_{s}^{*}$ at $r^{*}=R^{*}(z)$

$\tau^{*}$ is finite $r^{*}=0$

where $w_{s}^{*}$ is the axial slip velocity.

\subsection{Solution of the Problem}

Introducing the non-dimentional form

$p=\frac{p^{*}}{\rho W_{0}^{2}}, w=\frac{w^{*}}{W_{0}}, r=\frac{r^{*}}{R_{0}}, z=\frac{z^{*}}{R_{0}}, \tau=\frac{\tau^{*}}{\rho W_{0}^{2}}$

the governing system of Eqn's (2) become

$0=-C+\frac{1}{r} \frac{\partial}{\partial r}(r \tau)-\frac{M^{2}}{\operatorname{Re}} w$

where $M=\sqrt{\frac{\sigma}{\rho \gamma}} B_{0} R_{0}, \quad \operatorname{Re}=\frac{R_{0} W_{0}}{\gamma}, C=\frac{d p}{d z}$.

The boundary conditions given in Eq. (4) reduces to

$w=w_{s}$ at $r=R(z)$

$\tau$ is finite $r=0\}$

where $w_{s}$ is the axial slip velocity.

Using the boundary conditions given in Eq. (6) in Eq. (5) and assuming that the average velocity to be constant for incompressible fluid flow, we get

$$
\tau=-\frac{r}{2}\left(C+\frac{M^{2}}{\operatorname{Re}} W_{c}\right)
$$

and $\tau_{\mathrm{R}}=-\frac{\mathrm{R}}{2}\left(C+\frac{M^{2}}{\mathrm{Re}} W_{c}\right)$

where $\tau_{\mathrm{R}}$ is the wall shear stress and $R=R(z)$ and $W_{c}$ is the constant average velocity.

Now integrating Eq. (3) and using the boundary conditions given in Eq. (6), Eq. (7), Eq. (8) , the velocity function become
$w(r)=w_{s}+\frac{R(z)}{(n+1) k \tau_{R}}\left\{\left(\tau_{R}-\tau_{H}\right)^{n+1}-\left(\frac{r}{R} \tau_{R}-\tau_{H}\right)^{n+1}\right\}$

where

$$
r_{p} \leq r \leq R(z)
$$

The volumetric rate of flow is defined by

$$
\begin{aligned}
& Q=\int_{0}^{R(z)} 2 \pi r w(r) d r \\
& Q=\pi R(z)^{2} w_{s}+\frac{\pi R(z)^{3}}{(n+3) k} \tau_{R(z)}^{n}
\end{aligned}
$$$$
\left\{1+\left(\frac{2}{n+2}\right) \frac{\tau_{\mathrm{H}}}{\tau_{R(z)}}+\left(\frac{2}{(n+1)(n+2)}\right)\left(\frac{\tau_{\mathrm{H}}}{\tau_{R(z)}}\right)^{2}\right\}\left(1-\frac{\tau_{\mathrm{H}}}{\tau_{R(z)}}\right)^{n+1}
$$

\subsection{Results and Discussions}

The problem under investigation is solved analytically and the expressions derived have been computed for different parameters such as for Hartmann number, stenosis height, yield stress, Reynolds number. In this paper the effects of these parameters on axial velocity, volumetric flow rate and wall shear stress in presence of external magnetic field is studied. The present analysis corresponds to the flow of Newtonian fluid and that in a normal artery for parameter values $\mathrm{n}=1, \tau_{H}=0.0$ respectively.

It is observed from Fig. 1 that the axial velocity decreases for increasing value of the magnetic parameter i.e. the Hartmann number for fixed values of $\mathrm{z}=8, \mathrm{n}=1, \mathrm{k}=2, \mathrm{C}=1.46$ and slip velocity $w_{s}=0.05$. It is due to the fact that as the magnetic field applied, the Lorentz force opposes the flow of blood and hence reduces the velocity. Again for the same fixed values the axial velocity with radial distance decreases for increasing values of stenosis height and yield stress which is shown in the Fig. 2 and Fig. 3. Fig. 4 shows that the axial velocity increases for increasing values of Reynolds number.

The variation of blood flow rate with axial distance is shown in Fig. 5 and Fig. 6 for different values of Hartmann number and yield stress respectively. The graphical results shows that magnetic field and yield stress reduces the flow rate for the same fixed values $\mathrm{z}=8, \mathrm{n}=1, \mathrm{k}=2, \mathrm{C}=1.46$ and $w_{s}=0.05$. Also from Fig. 7 and Fig. 8 we have seen that the wall shear rate decreases with increasing value of Hartmann number and yield stress. 


\section{GRAPHICAL ANALYSIS}

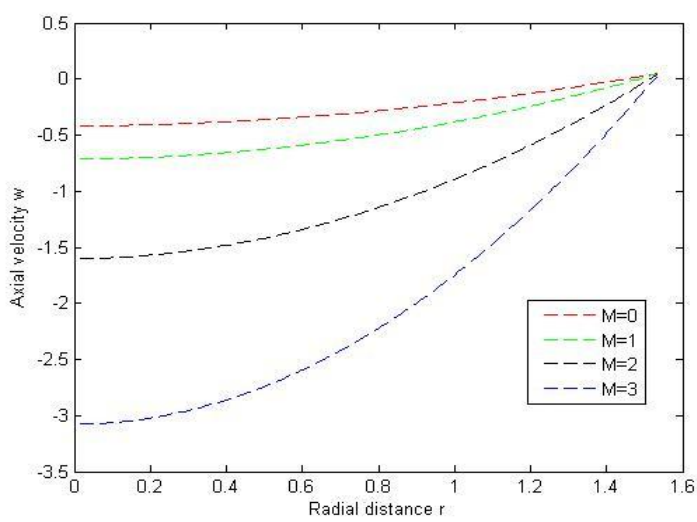

Fig 1: Variation of axial velocity with radial distance for different values of Hartmann number

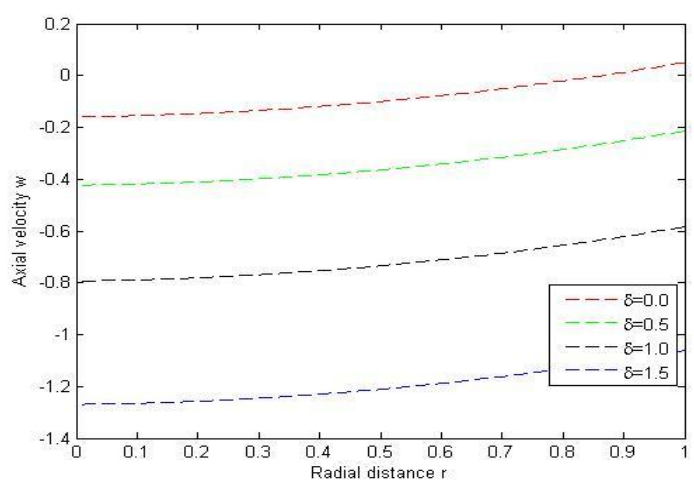

Fig 2: Variation of axial velocity with radial distance for different values of Stenosis height

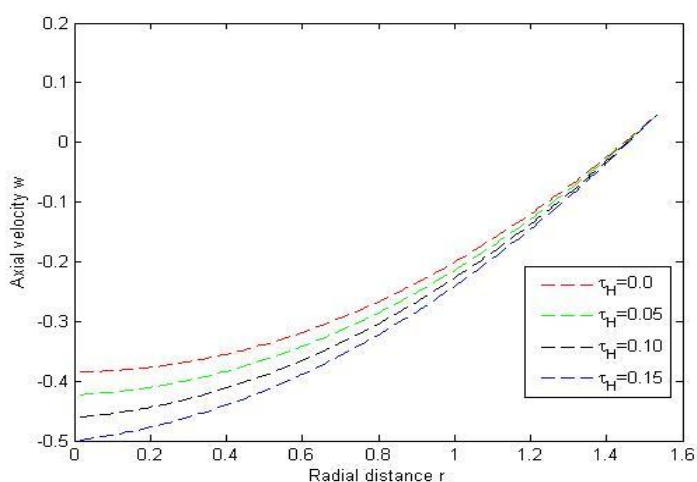

Fig 3: Variation of Axial velocity with radial distance for different values of yield stress

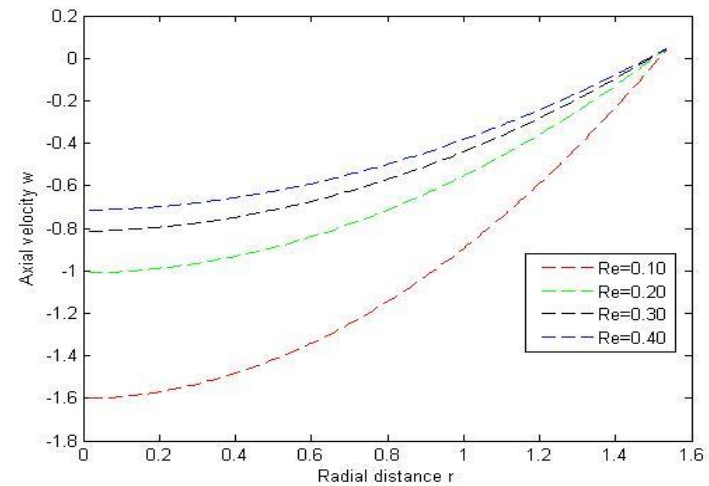

Fig 4: Variation of axial velocity with radial distance for different values of Reynolds Number

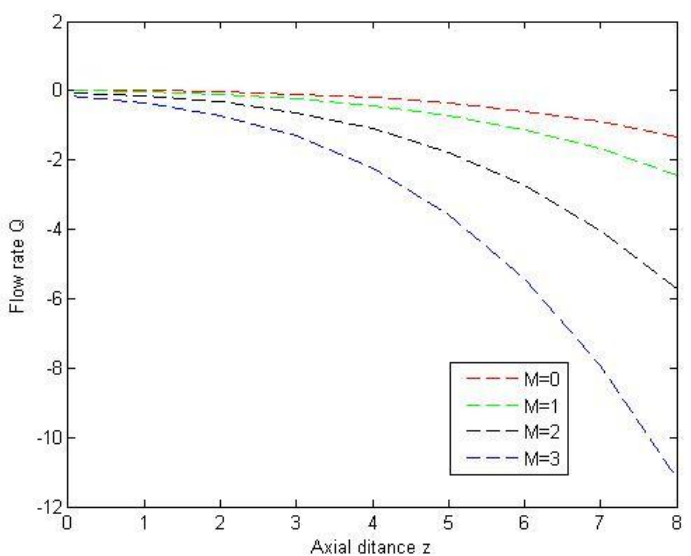

Fig 5:Variation of flow rate with axial distance for different values of Hartmann number

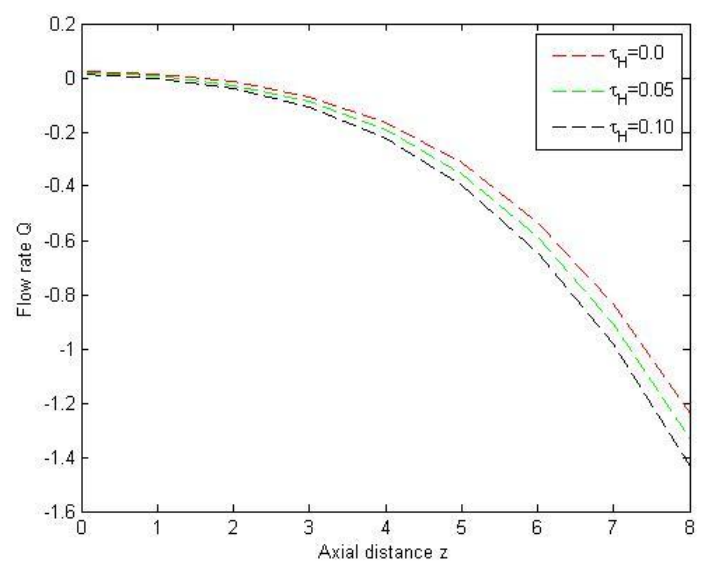

Fig 6: Variation of flow rate with axial distance for different values of yield Stress 


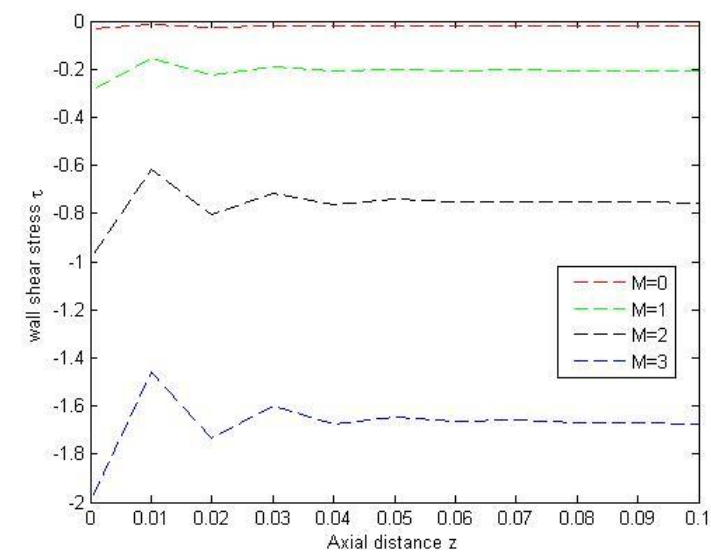

Fig 7: Variation of wall shear stress with axial distance for different values of Hartmann number

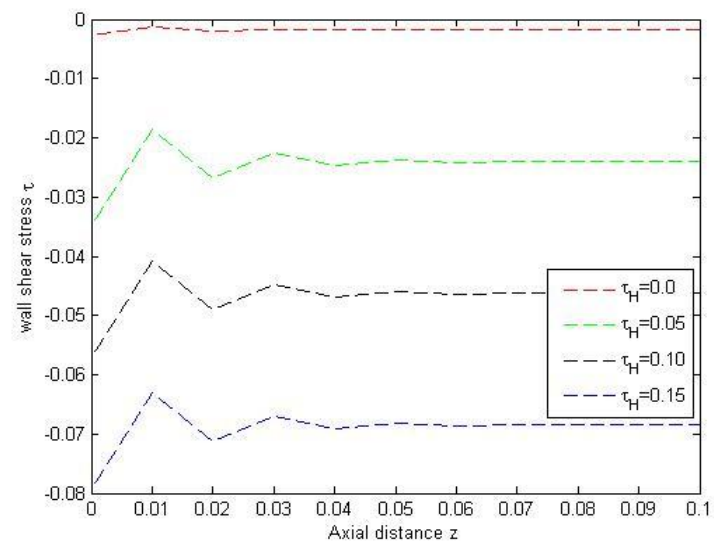

Fig 8: Variation of wall shear stress with axial distance for different values of yield stress

\section{CONCLUSION}

From the above analysis we can conclude that a significant variation takes place in flow characteristics due to nonNewtonian rheology and application of magnetic field. Hence the non-Newtonian behavior is an important factor and should not be neglected. It is seen that the flow rate and wall shear rate decreases with the increase in strength of Magnetic field.
So the present study would be helpful in blood pressure control. This present analysis also provides a scope to study the influence of induced magnetic field on different flow characteristics.

\section{REFERENCES}

[1] Fry, D. L., 1972 Localizing Factors in Arteriosclerosis. In: Arteriosclerosis and Coronary Heart Disease, Likoff, W., B.L. Segal and W. Jr.Insull(Eds.). Grune and Stratton, New York, pp.85-104

[2] Young, D.F. Tsai F.Y., 1973 Flow characteristics in models of arterial stenosis-II. unsteady flow .J.Biomech., 6:547-559.

[3] Lee, J.S., 1974 On the coupling and detection of motion between an artery with a localised lesion and its surrounding tissue.J.Biomech., 7 pp. 403-409.

[4] May, A.G., Deweese J.A., 1963 Rob C.G., Hemodynamic effects of arterial stenosis.Surgery, $53 \mathrm{pp}$. 513-524.

[5] Casson, N.A., 1959 Flow Equation for Pigment Oil Suspensions of the Printing Ink type. In:Rheology of Disperse Systems, Mill, C. C. (Ed.). Pergamon Press, London, pp. 84-102.

[6] Reiner M., ScottBaldair G.W., 1959 The flow of blood through narrow tube.Nature, 184:354-354.

[7] Sarma M., 2012 analysis of blood flow through stenosed vessel under Effect of magnetic fieldinternational journal for basic sciences and social sciences (3), pp. 78-88.

[8] Ikbal M.K, Chakravarty S, Wong K.L, Mazumdar J, Mandal P.K, 2009 Journal of Computational and Applied Mathematics, 230(1) pp. 243-259.

[9] Singh J, Rathee R (2011) Analysis of non-Newtonian blood flow through stenosed vessel in porous medium under the effect of magnetic field. Int. J. Phys. Sci. 6: 2497-2506.

[10] Biswas, D, and Laskar,R.B., 2011 Steady flow of Blood through Stenosed Artery :A Non-Newtonian Fluid Model ,Assam University J. of Sci. and Technology : physical Sci. and Technology, Vol. 7 (II) 144-153 